\title{
Composition chimique et valeur nutritionnelle pour les ruminants d'un ensilage de mycélium d'Aspergillus niger
}

\author{
A Théwis, P Petit \\ Faculté des sciences agronomiques, passage des Déportés, 2, 5030 Gembloux, Belgique
}

La production d'acide citrique par fermentation des mélasses au moyen d'Aspergillus niger donne, entre autres, un sous-produit solide : le mycélium.

Deux lots de ce mycélium sont ensilés dans 6 fûts métalliques. Trois échantillons sont prélevés par fût, regroupés et analysés pour les caractéristiques fermentaires et la composition chimique selon le schéma de Weende. On mesure, en plus, les teneurs en protéines réelles totales (PRT) par précipitation de celles-ci au moyen d'hydroxyde de cuivre, en acides aminés et en lignocellulose (ADF). Ca, Mg, Zn, Mn, Fe et Cu sont dosés par absorption atomique, $\mathrm{Na}$ et $\mathrm{K}$ par photométrie et $P$ par colorimétrie. La valeur énergétique du mycélium est calculée à partir des digestibilités mesurées par la méthode des différences sur 8 béliers recevant $40 \mathrm{~g} \mathrm{MS} / \mathrm{kgP}^{0,75}$ d'ensilage de maïs ou de ce même ensilage et de mycélium $(40 / 60 \mathrm{w} / \mathrm{w})$. La dégradabilité théorique de l'azote $(D T 0,06)$ est obtenue par la méthode d'Ørskov et McDonald (1979) après incubation des sachets de nylon dans le rumen de 3 bceufs recevant une ration composée de foin et concentré $(50 / 50)$ et distribuée à raison de $70 \mathrm{~g} \mathrm{MS} / \mathrm{kgP}^{0,75}$.

La conservation du mycélium par ensilage est bonne : $\mathrm{pH}: 4,5$, pertes par les jus : 2 à $4 \%$ de la matière fraîche, $20 \mathrm{~g}$ d'acide lactique et $25 \mathrm{~g}$ d'acide acétique par $\mathrm{kg}$ de MS. La teneur en MS de l'ensilage est élevée $(28,7 \%)$. La concentration en matières azotées totales dans la MS est de $34,2 \%$ dont environ $68 \%$ seulement sont consti- tués de PRT. La présence d'acides nucléiques et de chitine permet notamment d'expliquer la différence. Les acides aminés essentiels représentent $46 \%$ des acides aminés totaux. On note une teneur intéressante en tryptophane $(2,2 \mathrm{~g} / 16$ $\mathrm{g} \mathrm{N}$ ) mais assez faible en acides aminés soufrés $(2,1 \mathrm{~g} / 16 \mathrm{~g} \mathrm{~N})$ et en lysine $(2,7 \mathrm{~g} / 16 \mathrm{~g} \mathrm{~N})$. La teneur en extrait éthéré est élevée $(6,6 \%)$. La cellulose brute représente $18,8 \%$ de la MS et la concentration en lignocellulose est de $23,4 \%$. Ces teneurs élevées sont à mettre en relation avec la présence de chitine. La concentration en cendres totales est élevée $(14,1 \%)$. On relève aussi de fortes teneurs en Ca $(28,7 \mathrm{~g} / \mathrm{kg})$ et également en $\mathrm{K}(15,2 \mathrm{~g} / \mathrm{kg})$, Fe (1004 mg/kg) et $\mathrm{Zn}$ $(92,2 \mathrm{mg} / \mathrm{kg})$. En revanche, cet ensilage est pauvre en $\mathrm{P}(1,4 \mathrm{~g} / \mathrm{kg})$, $\mathrm{Na}(2,8 \mathrm{~g} / \mathrm{kg}), \mathrm{Mg}(0,6$ $\mathrm{g} / \mathrm{kg}), \mathrm{Cu}(8,4 \mathrm{mg} / \mathrm{kg})$ et $\mathrm{Mn}(42,1 \mathrm{mg} / \mathrm{kg})$.

L'ingestion de la ration d'ensilage de maïs et de mycélium n'a posé aucun problème particulier. Les digestibilités de la matière organique, des matières azotées totales, de la cellulose brute et de l'extrait éthéré sont respectivement de 0,77 , $0,86,0,76$ et 0,65 . II en résulte des teneurs en énergie nette de 960 VEM ou 0,99 UFL et 994 VEVI ou 0,94 UFV par $\mathrm{kg}$ de MS. La DT de l'azote est de 0,71. Les teneurs en PDI sont de $89 \mathrm{~g}$ de PDIA, $223 \mathrm{~g}$ de PDIN et $131 \mathrm{~g}$ de PDIE.

Ørskov ER, McDonald I (1979) J Agric Sci Cambridge 92, 499-503 